\title{
Specify Other Preoperative IV Antacids Administered
}

National Cancer Institute

\section{Source}

National Cancer Institute. Specify Other Preoperative IV Antacids Administered. NCI

Thesaurus. Code C162673.

A request to specify intravenous antacids that were administered preoperatively but not included on a list. 